\title{
Primary Tumor Volume Is an Independent Predictor of Outcome Within pT4a-Staged Tongue Carcinoma
}

\author{
Mu-Kuan Chen, MD, MS, PhD ${ }^{1,2,3}$, Chih-Ming Chen, MD ${ }^{1}$, Ming-Che Lee, MS ${ }^{4}$, Li-Sheng Chen, $\mathrm{PhD}^{5}$, \\ and Hui-Chuan Chen, $\mathbf{P h D}^{6}$ \\ ${ }^{1}$ Department of Otorhinolaryngology, Head and Neck Surgery, Changhua Christian Hospital, Changhua, Taiwan; \\ ${ }^{2}$ Mingdao University, Changhua, Taiwan; ${ }^{3}$ School of Medicine, Chung Shan Medical University, Taichung, Taiwan; \\ ${ }^{4}$ Department of Medical Imaging, Changhua Christian Hospital, Changhua, Taiwan; ${ }^{5}$ Laboratory of Biostatistics, \\ Changhua Christian Hospital, Changhua, Taiwan; ${ }^{6}$ Providence University, Taichung, Taiwan
}

\begin{abstract}
Background. We evaluated the heterogeneity of primary tumor volume (PTV) within tumors of the same pT4astaged tongue carcinoma and to elucidate the effects of PTV on treatment outcomes in patients with pT4a-staged tongue carcinoma.

Methods. Fifty-eight patients with newly diagnosed pT4astaged tongue carcinoma who received surgery were enrolled onto this study. Magnetic resonance imagingderived PTV was measured by the summation-of-area technique.
\end{abstract}

Results. The mean PTV was $24.55 \mathrm{ml}$, with a range of 5.32 to $119.64 \mathrm{ml}$. The receiver operating characteristic curve was applied, and the optimal cutoff volume was $23 \mathrm{ml}$. Large PTV was associated with a significantly poor disease-specific survival $(P=0.010)$ by the log rank test. The Cox regression model also revealed that large PTV $(P=0.026)$ and positive lymphatic node metastasis $(P=0.004)$ were statistically significant in the prognosis of T4a-staged tongue carcinoma.

Conclusions. A substantial variation of PTV was present within the same pT4a-staged tongue carcinoma, and PTV represented an important prognostic factor. In the light of

Part of this study was presented at the Second World Congress of the International Academic of Oral Oncology 8-11 July 2009, Toronto, Canada.

(C) The Author(s) 2010. This article is published with open access at Springerlink.com

First Received: 21 April 2010;

Published Online: 21 December 2010

M.-K. Chen, MD, MS, PhD

e-mail: 53780@cch.org.tw these findings, we suggest that taking the PTV into account in pT4a-staged tongue carcinoma would better refine the newest revised $\mathrm{T}$ classification, and the treatment strategies may be different.

Oral cancer is preponderant in Taiwan, with annual incidence rates of 37 per 100,000 for men. It also is ranked as the fourth leading cause of cancer death among men according to a 2005 cancer registry report. ${ }^{1}$ Regarding anatomic sites, tongue cancer is most common in Taiwan. As is the case for other malignancies, the staging system keeps changing and the identification of risk factors continues to develop for predicting the prognosis of patients and selecting appropriate treatment modalities. The most widely used system is the American joint Committee of Cancer (AJCC) staging system. ${ }^{2}$ Compared with other subsites of oral cancers, tongue cancer has some unique characteristics, as follows. The tongue consists of intrinsic and extrinsic muscular structures, so there is no strong barrier to stop the tumor from spreading. In addition, when compared to other head and neck anatomic site, the vascular and lymphatic systems of tongue are well developed, and thus the rate of cervical lymph node metastasis is high. ${ }^{3}$ Because of the unique anatomic structure of the tongue, there is much debate about the conventional T-stage classification that considers lesion size only. Since 1997, the AJCC has revised the $\mathrm{T}$ classification by incorporating extrinsic muscle invasion. ${ }^{2}$ However, the debates continue; for example, some authors have suggested that the depth of invasion was associated with neck metastasis and affected nodal upstaging in tongue cancer or head and neck cancer. ${ }^{4-6}$

Studies have shown that tumor volumes predicted local control of head and neck cancers. Large tumors were more likely to recur than small tumors in the same location. 
Threshold volumes for oropharyngeal, laryngeal, and pyriform sinus carcinomas have been published. ${ }^{7-9}$ In nasopharyngeal carcinoma, the impact of primary tumor volume has been well established by our team. ${ }^{10-15}$ To our knowledge, for the head and neck region, no literature has been published about the primary tumor volume for tongue cancer. Although such disease is resectable, clinicians commonly agree that the prognosis is poor in pT4a-staged tongue carcinoma, despite currently aggressive treatment. However, to our knowledge, the relationship between primary tumor volume and treatment outcome within the same pT4a-staged tongue carcinoma, which has the poorest prognosis, has never been studied.

The aims of this study were to evaluate the variability of primary tumor volume within the pT4a-staged tongue carcinoma and to elucidate the impact of primary tumor volume on the prognosis of patients with pT4a-staged tongue carcinoma after surgery and adjuvant therapy. The results may provide empiric evidence for refining both the tumor, node, metastasis system staging system and the clinical management of patients with pT4a-staged oral tongue carcinoma.

\section{PATIENTS AND METHODS}

\section{Patient Selection}

A sequential and prospectively maintained head and neck cancer database was retrospectively searched for newly diagnosed tongue carcinoma treated with surgery between 2003 and 2006. An institutional review board approved this study. During this 4-year period, 169 patients with newly diagnosed tongue cancer treated in our tertiary referral medical center and 153 patients (90.5\%) who underwent surgery with or without adjuvant chemoradiotherapy had complete data available for analysis. The inclusion criteria were as follows: primary oral tongue cancer; pathologically proven T4a(pT4a)-staged squamous cell carcinoma with preoperative magnetic resonance imaging (MRI); and accepted primary tumor wide excision with neck dissection in our medical center with definitely pathologic $\mathrm{T}$ and $\mathrm{N}$ classification. The exclusion criteria were as follows: presence of distant metastasis at the time of presentation (3 patients); artifacts on MRI that resulted in difficulty with delineating tumor contours (1 patient); and loss to follow-up (1 patient). Fifty-eight patients were eligible for analysis; 49 received postoperative radiotherapy and 32 received adjuvant chemotherapy. The end of follow-up was November 30, 2009.

\section{Tumor Volume Measurement}

All patients in this study underwent pretreatment MRI. The MRI was performed on a 1.5-T magnetic resonance unit (Magnetom Avanto, Siemens Medical Solution,
Erlangen, Germany). Sequences included axial T1-weighted images (TR/TE/NEX, 450/20/3; matrix size, $320 \times 80$; section thickness, $4 \mathrm{~mm}$ ), axial T2-weighted images (TR/ TE/NEX, 4520/76/3; matrix size, $384 \times 80$; section thickness, $4 \mathrm{~mm}$ ), axial fat-suppressed T2-weighted sequence (repetition time [TR]/echo time [TE], $5080 \mathrm{~ms} /$ $82 \mathrm{~ms}$; section thickness, $6 \mathrm{~mm}$; matrix size, $320 \times 80$ ), coronal fat-suppressed T2-weighted sequence (TR/TE, $4000 \mathrm{~ms} / 100 \mathrm{~ms}$; section thickness, $4 \mathrm{~mm}$; matrix size, $256 \times 90)$ and contrast-enhanced T1-weighted spin-echo images with the use of a $320 \times 80$ matrix in the axial planes and $320 \times 80$ matrix in coronal and sagittal planes after a bolus injection of $0.1 \mathrm{mmol} / \mathrm{kg}$ gadopentetate dimeglumine (Schering, Berlin, Germany). The T1-TEaxial images with contrast and fat suppression were selected for tumor volume measurements; the other sequences were taken to provide for auxiliary diagnosis. With applied software, each image of T1-TE-axial view with contrast plus fat suppression was visualized on a large-screen monitor. User-defined magnification of the images was possible. For tumor characteristics, clinicians kept track of the tumor contour manually. The primary tumor volume was calculated by the summation-of-area technique, which multiplies the entire areas by the image reconstruction interval of $4 \mathrm{~mm}$. As was done in our previous studies and also in a pilot study that focused on 10 patients with tongue cancer, in the current study, one author (C.M.C.) was the major assessor of tumor volumes and was assisted by the senior author (M.K.C.). ${ }^{10-15}$ Thus two clinicians read the MRIs and kept track of the tumor contours, and another author (C.M.C.) then calculated the tumor volume. A radiologist who specialized in head and neck malignancies participated when the judgments of the two authors differed; and the tumor contour was assessed by consensus. One patient was excluded from this study because of difficulty with delineating tumor contours by the three clinicians. To reduce bias, assessors of the tumor volumes were blinded to the patients' condition.

\section{Statistical Analysis}

To segregate the patients into large and small tumor volume groups, the receiver operating characteristic (ROC) analysis was applied to determine the optimal cutoff point value obtained by calculating the sensitivity and specificity of every observed data value and plotting sensitivity against 1 - specificity. Neck lymph node metastasis, pathological grade, lymphovascular invasion, perineural invasion, status of surgical margin, and cross midline extension were analyzed by univariate analysis. Diseasespecific survival was the primary outcome variable for this study. The survival curves were calculated by the KaplanMeier method, and differences were assessed by the log 
rank test. A Cox proportional hazard regression model also was applied to access the correlations between primary tumor volume and neck metastasis (with or without adjuvant chemotherapy and radiotherapy), and the prognosis of patients after they received therapy while controlling for age and sex. A $P$ value of $\leq 0.05$ was considered to be statistically significant.

\section{RESULTS}

A substantial variation in primary tumor volume was observed within the same T4a-staged disease. The mean primary tumor volume was $24.55 \mathrm{ml}$, with a range of 5.32 to $119.64 \mathrm{ml}$. The ROC curve revealed that the optimal cutoff volume was $23 \mathrm{ml}$; the area under the ROC curve was $68.5 \%$ (Fig. 1). Patients were followed from 5 to 80 months, and the mean follow-up time was 28.4 months. Patient characteristics, neck lymph node status, pathological grade, lymphovascular invasion, status of surgical margin, cross midline extension, follow-up, and the distribution of primary tumor volume are presented in Table 1. Only cross midline extension and the mean tumor volume were statistically associated with larger tumor volume $(P<0.05)$, whereas sex, age, neck lymph node status, pathological grade, perineural invasion, lymphovascular invasion, and status of surgical margin did not reach statistical significance. However, patients with large primary tumor volume had higher neck metastasis rate (68\% vs. $44 \%$ ), although this did not reach the statistical significance $(P=0.08)$. Figure 2 shows that after segregating the primary tumor volume into two subgroups $(<23 \mathrm{ml}$ and $\geq 23 \mathrm{ml})$, large primary tumor volume $(\geq 23 \mathrm{ml})$ was associated with a significantly poorer disease-specific survival $(P=0.010)$. The 2-year disease-

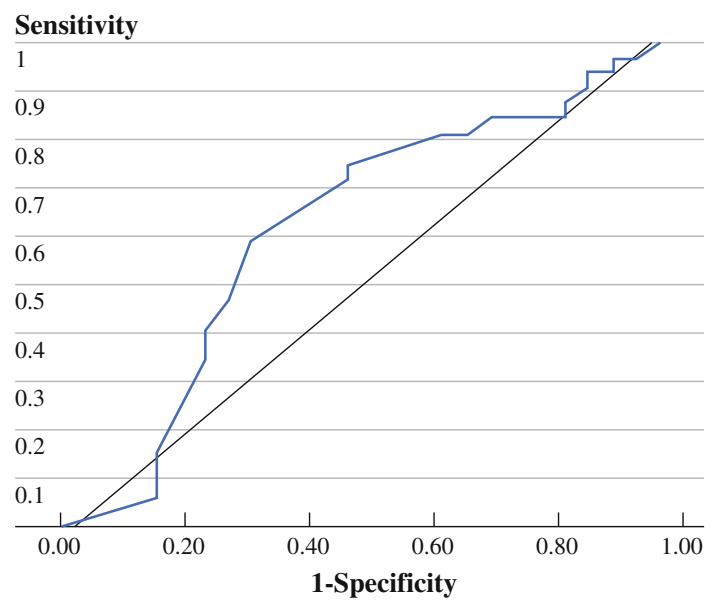

FIG. 1 Receiver operating characteristic curve for various cutoff values of tumor volume (area under curve $=68.5 \%$; optimal cutoff volume $=23 \mathrm{~mL}$ ) specific survival rates were $72 \%$ in patients with small tumor volumes versus $42 \%$ in patients with large tumor volumes within the same pT4a-staged disease. Six patients had local recurrence, and 5 of them $(83.3 \%)$ belonged to the large primary tumor volume. The Cox regression model revealed that the primary tumor volume $(P=0.026$; risk ratio $=2.68 ; 95 \%$ confidence interval $[95 \% \mathrm{CI}]$, 1.13-6.39) and neck lymph node metastasis $(P=0.003$; risk ratio $=7.13 ; 95 \% \mathrm{CI}, 1.94-25.18)$ were statistically significant in the prognosis of pT4a-staged tongue carcinoma, whereas pathological grade, postoperative radiotherapy, and postoperative chemotherapy were not statistically significant (Table 2).

\section{DISCUSSION}

Accurate disease staging of patients with malignancies is essential to divide patients into prognostic subgroups, to allow delivery of stage-specific therapies, and to facilitate meaningful discussions between physicians and patients regarding management and expected outcomes. The AJCC $\mathrm{T}$ classification of oral tongue carcinoma is mainly based on tumor size. In 2002, T classification was revised, adding the presence or absence of extrinsic muscle invasion as stage T4a. ${ }^{16}$ In oral cavity cancer, $\mathrm{T} 4 \mathrm{a}$ is defined as tumor invades adjacent structures (e.g., through cortical bone, into deep [extrinsic] muscle of tongue [genioglossus, hyoglossus, palatoglossus, and styloglossus]), maxillary sinus, and skin of face. In this study, we used this T classification and selected the pT4a-staged patients to be our target population. Figure 1 shows that large primary tumor volume was associated with a significantly poorer diseasespecific survival within the same pT4a-staged tongue carcinoma. This result revealed that taking the primary tumor volume into account in T4a-staged tongue carcinoma would better refine the newest revised $\mathrm{T}$ classification.

To investigate the association between the primary tumor volume and other factors, including sex, age, neck lymph node status, pathological grade, cross midline extension, perineural invasion, lymphovascular invasion, and status of surgical margin, we performed univariate analysis (Table 1). The results showed that only cross midline extension reaches statistical significance, while large tumor volume had the tendency to neck lymph node metastasis, high pathological grade, and lymphovascular invasion. Because of our study's small sample size, we did not input all variables in the Cox regression model; the results revealed that primary tumor volume $(P=0.026$; risk ratio $=2.68 ; 95 \% \mathrm{CI}, 1.13-6.39)$ and neck lymph node metastasis $(P=0.003$; risk ratio $=7.13 ; 95 \% \mathrm{CI}$, 1.94-25.18) were statistically significant in the prognosis of pT4a-staged tongue carcinoma. Thus, we suggest that 
TABLE 1 Characteristics of 58 patients with T4a-staged tongue carcinoma

\begin{tabular}{|c|c|c|c|c|}
\hline \multirow[t]{2}{*}{ Characteristic } & \multicolumn{2}{|c|}{ Primary tumor volume } & \multirow[t]{2}{*}{ Total patients } & \multirow[t]{2}{*}{$P$} \\
\hline & $<23 \mathrm{ml}$ & $\geq 23 \mathrm{ml}$ & & \\
\hline No. of patients & 36 & 22 & 58 & \\
\hline \multicolumn{5}{|l|}{ Sex, $n(\%)$} \\
\hline Female & $4(11 \%)$ & $0(0 \%)$ & $4(7 \%)$ & 0.2868 \\
\hline Male & $32(89 \%)$ & $22(100 \%)$ & $54(93 \%)$ & \\
\hline Age $(y)($ mean \pm SD) & $52.27 \pm 12.61$ & $48.04 \pm 7.65$ & $50.67 \pm 11.12$ & 0.1613 \\
\hline \multicolumn{5}{|l|}{ Lymph node, $n(\%)$} \\
\hline Negative & $20(56 \%)$ & $7(32 \%)$ & $27(47 \%)$ & 0.0787 \\
\hline Positive & $16(44 \%)$ & $15(68 \%)$ & $31(53 \%)$ & \\
\hline \multicolumn{5}{|l|}{ Pathological grade, $n(\%)$} \\
\hline Well & $5(14 \%)$ & $3(14 \%)$ & $8(14 \%)$ & 0.8877 \\
\hline Moderate & $29(80 \%)$ & $17(77 \%)$ & $46(79 \%)$ & \\
\hline Poorly & $2(6 \%)$ & $2(9 \%)$ & $4(7 \%)$ & \\
\hline \multicolumn{5}{|l|}{ Perineural invasion, $n(\%)$} \\
\hline Negative & $19(53 \%)$ & $13(59 \%)$ & $32(55 \%)$ & 0.6390 \\
\hline Positive & $17(47 \%)$ & $9(41 \%)$ & $26(45 \%)$ & \\
\hline \multicolumn{5}{|l|}{ Lymphovascular invasion, $n(\%)$} \\
\hline Negative & $31(86 \%)$ & $17(77 \%)$ & $48(55 \%)$ & 0.3872 \\
\hline Positive & $5(14 \%)$ & $5(23 \%)$ & $10(45 \%)$ & \\
\hline \multicolumn{5}{|l|}{ Cross midline, $n(\%)$} \\
\hline Negative & $34(94 \%)$ & $6(27 \%)$ & $40(69 \%)$ & $<0.001$ \\
\hline Positive & $2(7 \%)$ & $16(73 \%)$ & $18(31 \%)$ & \\
\hline \multicolumn{5}{|l|}{ Surgical margin, $n(\%)$} \\
\hline Negative & $35(97 \%)$ & $22(100 \%)$ & $57(98 \%)$ & 1.000 \\
\hline Positive & $1(3 \%)$ & $0(0 \%)$ & $1(2 \%)$ & \\
\hline Tumor volume $(\mathrm{ml})($ mean $\pm \mathrm{SD})$ & $12.57 \pm 5.56$ & $44.14 \pm 26.76$ & $24.55 \pm 2.84$ & $<0.0001$ \\
\hline Follow-up time $(\mathrm{mo})($ mean $\pm \mathrm{SD})$ & $31.30 \pm 18.41$ & $22.04 \pm 14.73$ & $27.79 \pm 18.45$ & 0.0632 \\
\hline
\end{tabular}

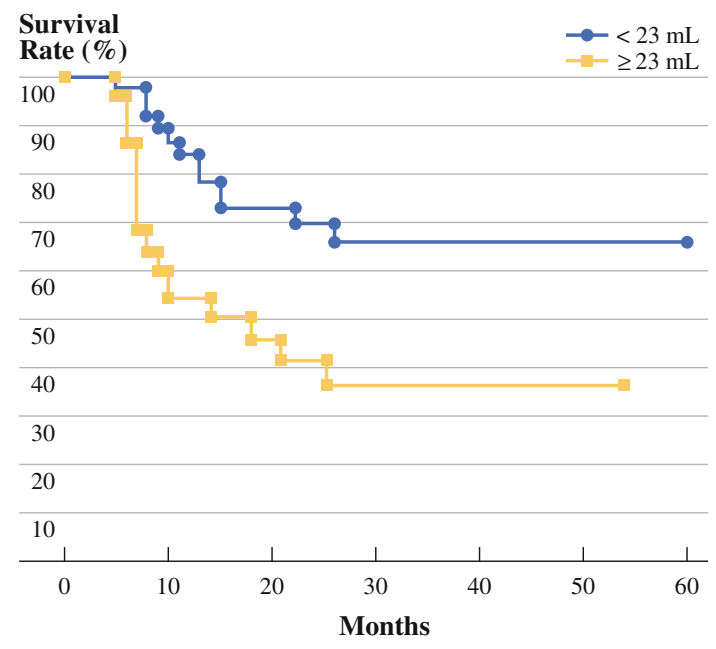

FIG. 2 Disease-specific survival curves of 58 T4a-staged tongue carcinoma according to large $(\geq 23 \mathrm{~mL})$ and small $(<23 \mathrm{~mL})$ tumor volumes. Within the same T4a-satge, large primary tumor volume was associated with a significantly poor disease specific survival $(P=.0104)$

T4a tongue cancer with large primary tumor volume $(\geq 23 \mathrm{ml})$ and neck metastasis should be treated more aggressively.
TABLE 2 Cox regression model for prognosis of T4a-staged tongue carcinoma

\begin{tabular}{llll}
\hline Variable & $\mathrm{RR}$ & $95 \% \mathrm{CI}$ & $P$ \\
\hline $\begin{array}{l}\text { Primary tumor volume (ml) } \\
\quad 23 \mathrm{ml} \text { vs. }<23 \mathrm{ml}\end{array}$ & 2.68 & $1.13-6.39$ & $0.0261^{*}$ \\
$\begin{array}{l}\text { Neck node metastasis } \\
\quad \text { Positive vs. negative }\end{array}$ & 6.92 & $1.86-25.72$ & $0.0039^{*}$ \\
$\quad \begin{array}{l}\text { Pathological grade } \\
\text { Radiotherapy }\end{array}$ & 1.25 & $1.31-5.00$ & 0.7490 \\
$\quad$ With vs. without & 1.17 & $0.21-6.85$ & 0.8488 \\
$\begin{array}{l}\text { Chemotherapy } \\
\text { With vs. without }\end{array}$ & 1.16 & $0.25-5.32$ & 0.8521 \\
\hline
\end{tabular}

$R R$ risk ratio for disease-related survival, $95 \%$ CI $95 \%$ confidence interval

Adjusted for age and sex

$* P<0.05$

The result of surgery with postoperative chemoradiotherapy for advanced tongue carcinoma has improved over the years; however, it still remains unsatisfactory. In this study, the prognosis of patients with primary tumor 
volumes of $>23 \mathrm{ml}$ was poor; the 2-year survival rate was only $42 \%$. We suggest that the new technique of radiotherapy, the combination of different regimens of chemotherapy, and the use of targeted chemotherapy or immunotherapy may be useful for these patients. Also, for such patients, postoperative swallowing and phonation function are affected. The conventional wisdom that surgery is the mainstay of treatment should be challenged.

The current study has some limitations. First, although we did our best to estimate the tumor volume, the obtained tumor volume is still not the true tumor volume. Some error in estimating the tumor volume remains. However, our previous study revealed that if a single trained observer estimated the tumor volume, the information bias would be toward the null. ${ }^{10-15}$ Thus, in the current study, we delineated the tumor contour by the consensus of two or three well-trained head and neck oncologists or radiologists, not independently. However, with the advancement of MRI and applied software, we believe the tumor volume might be measured not only manually, but also by well-designed software in the future; at that future time, we suggest that two separate clinicians independently review the MRIs, but not by consensus. In addition, we believe that if there is no well-trained head and neck oncologist with experience measuring tumor volume, then the tumor volume measurements should be performed by a radiologist. ${ }^{10-15}$ Second, in the Cox regression model, because the radiotherapy dose is high, we neglected the fact that there was a relatively narrow range of dose, and we did not investigate the effect of total radiation dose on treatment outcome, which may obscure the important dose-response effect addressed by Willner et al. ${ }^{17}$ Thus, the total dose of radiotherapy still should be emphasized. Third, some authors have suggested that the depth of invasion was associated with neck metastasis and affected nodal upstaging in tongue cancer or head and neck cancer. ${ }^{4-6}$ In this study, we focused on the primary tumor volume only because we think that the primary tumor volume is threedimensional; it should be more meaningful than the conventional measures of tumor length or thickness. Thus, we did not evaluate the tumor thickness. Last, changes or revisions of any staging system should ideally be supported by evidence-based information generated from large cohorts of patients. Unfortunately, the head and neck region comprises a variety of anatomic sites, and these tumors have diverse clinical behavior and outcomes. It is therefore literally impossible to generate a uniform staging system that would be relevant for all tumors arising in the head and neck region. Another effect of this diversity among head and neck tumors is that patient cohorts available for outcomes analysis for most sites are limited in numbers, and on the whole, no level 1 evidence is available to support important clinical questions. ${ }^{18}$ In this study, we faced the same problem. The study was a retrospective one that searched a sequential and prospectively maintained head and neck cancer database with a limited number of patients. Although the treatment modality should follow our clinical guidelines, as a result of the patients' performance status or compliance, there was still bias in this study. Thus, we applied a Cox proportional hazard regression model to access the correlations between tumor volume, neck metastasis, presence or absence of chemotherapy and radiotherapy, pathological grade, and the prognosis of patients after they received therapy while controlling for age and sex. If a large patient cohort is available for meaningful data analysis, a prospective randomized trial should be started in the future.

To our knowledge, this study is the first to describe the primary tumor volume of oral tongue cancer. Primary tumor volumes in patients with tongue carcinoma were heterogeneous within the concurrent pT4a stage. Primary tumor volume represented an important prognostic factor for treatment outcome. We suggest that taking the primary tumor volume into account in pT4a-staged tongue carcinoma would better refine the newest revised $\mathrm{T}$ classification. Patients with large primary tumor $(\geq 23 \mathrm{ml})$ had poor prognosis and need more aggressive treatment.

CONFLICT OF INTEREST The authors declare no conflict of interest.

OPEN ACCESS This article is distributed under the terms of the Creative Commons Attribution Noncommercial License which permits any noncommercial use, distribution, and reproduction in any medium, provided the original author(s) and source are credited.

\section{REFERENCES}

1. Department of Health. Cancer Registry Annual Report Republic of China, 2005. Department of Health, The Executive Yuan, Republic of China, 2008.

2. Fleming ID, Cooper TS, Murphy GP, et al., editors. AJCC Cancer Staging Manual, 5th ed. Philadelphia: Lippincott-Raven, 1997.

3. Kim SH, Cho NH, Kim K, et al. Correlation of oral tongue cancer invasion with matrix metalloproteinase and vascular endothelial growth factor expression. J Surg Oncol. 2002;93:330-7.

4. Fukano H, Matsuura H, Hasegawa Y, Nakamura S. Depth of invasion as a predictive factor for cervical lymph node metastasis in tongue carcinoma. Head Neck. 1997;19:205-10.

5. Asakage T, Yokose T, Mukai K, et al. Tumor thickness predicts cervical metastasis in patients with stage I/II carcinoma of the tongue. Cancer. 1998;82:1443-8.

6. A lkureishi LW, Ross GL, Shoaib T, et al. Does tumor depth affect nodal upstaging in squamous cell carcinoma of the head and neck? Laryngoscope. 2008;118:629-34.

7. Pameijer FA, Mancuso AA, Mendenhall WM, Parsons JT, Kubilis PS. Can pretreatment computed tomography predict local control in T3 squamous cell carcinoma of the glottic larynx treated with definitive radiotherapy? Int J Radiat Oncol Biol Phys. 1997;37:1011-21. 
8. Johnson CR. Thames HD, Huang DT, Schmidt-Ullrich RK. The tumor volume and clonogen number relationship: tumor control predictions based upon tumor volume estimates derived from computed tomography. Int $J$ Radiat Oncol Biol Phys. 1995;33:281-8.

9. Pameijer FA, Mancuso AA, Mendenhall WM, et al. Evaluation of pretreatment computed tomography as a predictor of local control in $\mathrm{T} 1 / \mathrm{T} 2$ pyriform sinus carcinoma treated with definitive radiotherapy. Head Neck. 1998;20:159-68.

10. Chang CC, Chen MK, Liu MT, Wen YS, Wu HK. Primary tumor volume delineation in nasopharyngeal carcinoma and correlation with 1997 AJCC T-stage classification. J Otolaryngol. 2001;30:231-4.

11. Chang CC, Chen MK, Liu MT, Wu HK. Nasopharyngeal carcinoma volume measurements determined with computed tomography: study of intra-observer and inter-observer variability. J Otolaryngol. 2002;31:361-5.

12. Chang CC, Chen MK, Liu MT, Wu HK. The effect of primary tumor volumes in advanced T-staged nasopharyngeal carcinoma. Head Neck. 2002;24:940-6.
13. Chen MK, Lee HS, Chang CC. Primary tumor volume: important predictor of outcome for T3- and T4-staged nasopharyngeal carcinoma. J Otolaryngol. 2004;33:254-7.

14. Chen Mk, Lee HS, Chang JH, Chang CC. Expression of p53 protein and primary tumor volume in patients with nasopharyngeal carcinoma. J Otolaryngol. 2004;33:304-7.

15. Chen Mk, Chen THH, Liu JP, Chang CC, Chie WC. Better prediction of prognosis for patients with nasopharyngeal carcinoma using primary tumor volume. Cancer. 2004;100:2160-6.

16. Greene FL, Page DL, Fleming ID, et al., editors. AJCC cancer staging manual, 6th ed. New York: Springer, 2002.

17. Willner J, Baier K, Pfreundner L, Flentje M. Tumor volume and local control in primary radiotherapy of nasopharyngeal carcinoma. Acta Oncologica. 1999;38:1025-30.

18. Patel SG, Shah JP. TNM staging of cancers of the head and neck: striving for uniformity among diversity. CA Cancer J Clin. 2005;55:242-58. 